\title{
Downregulation of PRRX1 via the p53-dependent signaling pathway predicts poor prognosis in hepatocellular carcinoma
}

\author{
MINGMING FAN ${ }^{1 *}$, JUN SHEN $^{2 *}$, HU LIU $^{1 *}$, ZHIJIAN WEN $^{3 *}$, JUE YANG $^{1}$, \\ PINGHUA YANG ${ }^{1}, \mathrm{KAI} \mathrm{LIU}^{1}$, YANXIN CHANG ${ }^{1}, \mathrm{JICHENG} \mathrm{DUAN}^{1}$ and KAI LU ${ }^{1}$
}

\author{
${ }^{1}$ Department of Laparoscopy, Eastern Hepatobiliary Surgery Hospital, Second Military Medical University, Shanghai; \\ ${ }^{2}$ The First Department of Hepatic Surgery, Eastern Hepatobiliary Surgery Hospital, Second Military Medical University, \\ Shanghai; ${ }^{3}$ Department of Hepatobiliary Pancreatic Vascular Surgery, No. 174 Hospital of PLA, Xiamen, Fujian, P.R. China
}

Received January 6, 2017; Accepted June 1, 2017

DOI: $10.3892 / o r .2017 .5785$

\begin{abstract}
Paired-related homeobox 1 (PRRX1) has been identified as a novel molecule associated with induction of epithelial-mesenchymal transition (EMT), acquisition of cancer stem cell like properties and poor prognosis in tumors. However, the function of PRRX1 in hepatocellular carcinoma has not been elucidated. In the present study, we observed that PRRX1 expression levels were downregulated and positively correlated with the downregulated expression of p53 in hepatocellular carcinoma specimens. Decreased expression of PRRX1 and/or 153 by siRNA induced both the migration and the invasion features of HCC cells in vitro. Furthermore, the loss of PRRX1 inhibits hepatocellular carcinoma (HCC) cell apoptosis, an anti-apoptotic expression profile was upregulated accompanied by downregulated expression of p53. HCC patients with low-expression of both PRRX1 and p53 had a significantly shorter overall and disease-free survival. These findings demonstrate that PRRX1 plays an important role in metastasis and apoptosis of HCC cells through the p53-dependent signaling pathway and is expected to become a novel biomarker associated with patient prognosis and survival.
\end{abstract}

\section{Introduction}

Hepatocellular carcinoma (HCC) is the fifth most common cancer worldwide which represents more than $90 \%$ of primary liver cancers and is a major global health problem in excess of one million cases every year $(1,2)$. Despite the fact that surgical operation has made great progress during the past decades, patients with HCC still suffer a high incidence of postop-

Correspondence to: Dr Kai Lu, Department of Laparoscopy, Eastern Hepatobiliary Surgery Hospital, Second Military Medical University, 225 Changhai Road, Shanghai, 200438, P.R. China

E-mail: kailu_ehbh@163.com

*Contributed equally

Key words: PRRX1, p53, prognosis, hepatocellular carcinoma erative recurrence and metastasis. Therefore, it is necessary to investigate the molecular pathogenesis of HCC to develop novel treatment strategies.

Increasing evidence suggested that metastasis is initiated by epithelial-mesenchymal transition (EMT) at the invasive front of primary carcinoma $(3,4)$. EMT is recognized as an important step in invasion and metastasis which could be induced by cytokines $(5,6)$, transcription factors $(7,8)$ and other factors $(9,10)$. Paired-related homeobox 1 (PRRX1) was recently identified as a new EMT inducer (11). Furthermore, aberrant expression of PRRX1 is significantly associated with poor prognosis in various solid tumors including breast (11), colorectal (12), gastric cancer (13) and HCC (14). High PRRX1 expression levels were significantly associated with reduced metastasis and good prognosis in breast cancer (11), but the opposite relationship was observed in colorectal cancer and gastric cancer $(12,13)$. Downregulation of PRRX1 expression contributed to the poor prognosis of patients with breast cancer and HCC through acquisition of CSC-like properties $(11,14)$. However, the direct mechanisms through which PRRX1 regulates HCC cells is still unclear.

The tumor suppressor p53 is one of the most frequently mutated genes in human cancers that regulates the expression of stress response genes and mediates a variety of anti-proliferative processes $(15,16)$. Previous studies have shown that deletions or mutations of p53 are frequently found in cancers $(16,17)$ and that $\mathrm{p} 53$ is involved in tumor metastasis as well as tumor progression (18-20). In the present study, we investigated the expression of PRRX1 and p53 in HCC cells and clinical samples. We also found that aberrant expression of PRRX1 affect biological behavior of HCC cells by regulating p53. Finally, decreased expression of PRRX1 and p53 in $\mathrm{HCC}$ tissues is associated with poor prognosis.

\section{Materials and methods}

Patients and tissue specimens. Samples for the laboratory investigations were collected from April 2006 until February 2008. Formalin-fixed paraffin-embedded tumor tissues and matched adjacent non-tumorous hepatic tissues were collected from 116 HCC patients who underwent hepatectomy as an initial treatment at Eastern Hepatobiliary Surgery Hospital. 
Table I. Relationship between PRRX1 and p53 expression and clinicopathological features $(\mathrm{n}=116)$.

\begin{tabular}{|c|c|c|c|c|c|c|}
\hline \multirow[b]{2}{*}{ Variables } & \multicolumn{2}{|c|}{ PRRX1 expression } & \multirow[b]{2}{*}{ P-value ${ }^{a}$} & \multicolumn{2}{|c|}{ p53 expression } & \multirow[b]{2}{*}{ P-value ${ }^{a}$} \\
\hline & Low $(\mathrm{n}=77)$ & $\operatorname{High}(\mathrm{n}=39)$ & & Low $(n=45)$ & $\operatorname{High}(\mathrm{n}=71)$ & \\
\hline Sex & & & 0.553 & & & 0.290 \\
\hline Male & 47 & 26 & & 31 & 42 & \\
\hline Female & 30 & 13 & & 14 & 29 & \\
\hline Age (years) & & & 0.213 & & & 0.934 \\
\hline$\leq 50$ & 40 & 25 & & 25 & 40 & \\
\hline$>50$ & 37 & 14 & & 20 & 31 & \\
\hline Tumor size $(\mathrm{cm})$ & & & 0.91 & & & 0.582 \\
\hline$\leq 5$ & 58 & 29 & & 35 & 52 & \\
\hline$>5$ & 19 & 10 & & 10 & 19 & \\
\hline Serum AFP (ng/ml) & & & 0.209 & & & 0.582 \\
\hline$\leq 20$ & 45 & 18 & & 23 & 40 & \\
\hline$>20$ & 32 & 21 & & 22 & 31 & \\
\hline HBsAg & & & 0.724 & & & 0.661 \\
\hline Positive & 67 & 33 & & 38 & 62 & \\
\hline Negative & 10 & 6 & & 7 & 9 & \\
\hline Anti-HCV & & & 0.988 & & & 0.778 \\
\hline Positive & 4 & 2 & & 2 & 4 & \\
\hline Negative & 73 & 37 & & 43 & 67 & \\
\hline Liver cirrhosis & & & 0.507 & & & 0.903 \\
\hline Yes & 65 & 31 & & 37 & 59 & \\
\hline No & 12 & 8 & & 8 & 12 & \\
\hline Vascular invasion & & & 0.001 & & & 0.001 \\
\hline Yes & 53 & 11 & & 34 & 30 & \\
\hline No & 24 & 28 & & 11 & 41 & \\
\hline Intrahepatic metastasis & & & 0.002 & & & 0.001 \\
\hline Yes & 56 & 17 & & 20 & 53 & \\
\hline No & 21 & 22 & & 25 & 18 & \\
\hline Distant metastasis & & & 0.001 & & & 0.004 \\
\hline Yes & 49 & 4 & & 28 & 25 & \\
\hline No & 28 & 35 & & 17 & 46 & \\
\hline TNM stage & & & 0.036 & & & 0.005 \\
\hline I-II & 51 & 33 & & 22 & 62 & \\
\hline III-IV & 26 & 6 & & 23 & 9 & \\
\hline BCLC stage & & & 0.013 & & & 0.001 \\
\hline $0-\mathrm{A}$ & 62 & 23 & & 41 & 44 & \\
\hline $\mathrm{B}-\mathrm{C}$ & 15 & 16 & & 4 & 27 & \\
\hline
\end{tabular}

${ }^{\mathrm{a} C h i}$-square or Fisher's exact test.

For each patient, the diagnosis of HCC was confirmed on the basis of postoperative pathology (Fig. 1, representative pathohistological image). Preoperatively, no neoadjuvant radio- or chemotherapy was applied, and no invasive interventions, such as percutaneous ablation or chemo-embolization were performed. Each patient was followed up until March 2015. The Hospital Research Ethics Committee approved the research protocol. Written informed consents and voluntary participation in the study were obtained from every patient before the surgery. The clinical baseline characteristics of the HCC patients are presented in Table I.

Cell culture. The normal liver cell line LO2 and human HCC cell lines Hep3B, Huh7, HepG2, SMMC7721 (purchased from the Cell Bank of the Institute of Biochemistry and Cell Biology, Chinese Academy of Sciences, Shanghai, China) were 
cultured in Dulbecco's modified Eagle's medium (DMEM), supplemented with $10 \%$ fetal bovine serum (FBS; Gibco, Carlsbad, CA, USA), in humidified $5 \% \mathrm{CO}_{2}, 95 \%$ air at $37^{\circ} \mathrm{C}$.

Immunohistochemistry (IHC). The paraffin-embedded tissue specimens were cut into $4-\mu \mathrm{m}$ serial sections and placed on polylysine-coated slides. After deparaffinization in xylene, sections were rehydrated using a series of graded alcohols and microwave antigen retrievals. Slides were incubated in monoclonal antibodies against goat polyclonal anti-PRRX1 (NBP1-06067, 1:50 dilutions; Novus Biologicals LLC, Littleton, CO, USA), rabbit monoclonal anti-p53 (ab179477, 1:100 dilutions; Abcam, Cambridge, UK) at $4^{\circ} \mathrm{C}$ overnight, followed by incubation in the corresponding secondary antibodies at $37^{\circ} \mathrm{C}$ for $30 \mathrm{~min}$. Staining was performed with $\mathrm{DAB}$ and counterstaining with Mayer's hematoxylin. Negative controls were performed by omitting the primary antibodies.

To evaluate the expression of PRRX1 and p53, tissue sections were examined under a microscope at a magnification of $\times 200$. Ten fields were randomly selected to count tumor cells and to calculate the percentage of tumor cells with a stronger PRRX1 and p53 expression. In order to quantify the gene expression level, we created a score based on two criteria: i) the intensity of PRRX1 and p53 staining classified according to the following scale: negative, 0 ; weak, 1 ; and strong, 2. ii) The percentage of immunoreactive tumor cells was calculated and classified on a 5-point scale $(0,0 \%, 1,1-25 \%, 2,26-50 \%, 3$, $51-75 \%$, and 4, 76-100\%). For statistical analysis, a final score of 0-1 indicates low gene expression; a score of 2-4 indicates high expression of PRRX1 and p53.

Western blot analysis. Proteins from clinical specimens and HCC cell lines were extracted with lysis buffer (Beyotime Institute of Biotechnology, Haimen, China). Tissues and cell lysates were subjected to 10\% PAGE and transferred to nitrocellulose filter membranes. The membranes were blocked for $1 \mathrm{~h}$ in $5 \%$ non-fat dry milk diluted with TBST $(10 \mathrm{mM}$ Tris- $\mathrm{HCl}$ and $0.05 \%$ Tween-20). The membranes were then incubated with primary antibodies at $4^{\circ} \mathrm{C}$ overnight, followed by incubation with appropriate secondary antibodies at room temperature for $2 \mathrm{~h}$. The primary antibodies were goat polyclonal anti-PRRX1 (NBP1-06067, 1:500 dilutions; Novus Biologicals), rabbit monoclonal anti-p53 (ab179477, 1:10,000 dilutions; Abcam), mouse monoclonal anti-caspase-3 (ab2171, 1:500 dilutions; Abcam), rabbit polyclonal anti-Bax (ab7977, 1:1,000 dilutions; Abcam), mouse monoclonal anti-Bcl2 (ab117115, 1:1,000 dilutions; Abcam), and mouse monoclonal anti-GAPDH (sc-365062, 1:5,000 dilutions; Santa Cruz Biotechnology, Santa Cruz, CA, USA). The membranes were washed three times with phosphate-buffered saline (PBS), and the immunoreactive bands were visualized using an ECL Plus kit, according to the manufacturer's instructions. GAPDH was used as a gel loading control.

Small interfering RNA (siRNA) and transient transfection. PRRX1 siRNA was purchased from Santa Cruz Biotechnology (sc-106455). A non-functional siRNA (scrambled sequence) was used as control. p53 siRNA was purchased from Santa Cruz Biotechnology (sc-29435). The siRNA transfection was optimized using Lipofectamine 3000 (Invitrogen, Carlsbad,
CA, USA) according to the manufacturer's instructions; 24-48 $\mathrm{h}$ after the transfection, cells were analyzed using the assays described below.

Detection of cell migration and invasion ability. SMMC7721 and HepG2 were cultured and transfected with PRRX1 siRNA. The scrambled siRNA was used as control group, the parental cells were cultured at the same time as a blank control. Cells were added to the top chamber of Transwell plate $\left(3 \times 10^{5}\right.$ cells $\left./ 200 \mu \mathrm{l}\right)$. Normal medium (500 $\left.\mu \mathrm{l}\right)$ containing $10 \%$ FBS was added to the bottom chamber. When we detected cell invasion ability, Matrigel was plated to the top chamber. After culture for $48 \mathrm{~h}$, cells in the top chamber were removed and stained with $0.1 \%$ crytal violet for $15 \mathrm{~min}$. Ten fields were randomly imaged using the light microscope for counting. The experiment was repeated three times.

Wound healing assays and Transwell assays. For wound healing assays, cells were seeded in 6-well plates to a confluency of $\sim 60-70 \%$. Wounds were created in the cell monolayer with a $200-\mu 1$ pipette tip and the indicated plasmids were transfected into cells. Dead cells were eliminated with PBS wash. Wound closure was monitored at 0 and $24 \mathrm{~h}$. Cell invasion assays were evaluated using Transwell chamber assay (Millipore, Billerica, MA, USA) according to the manufacturer's instruction. Matrigel (BD Biosciences, San Jose, CA USA) was left to polymerize at the base of the top chamber of a 24-well Transwell plate ( $8 \mu \mathrm{m}$; Corning Costar Corp., Corning, NY, USA) for $45 \mathrm{~min}$ at $37^{\circ} \mathrm{C}$. Cells $\left(5 \times 10^{4}\right.$ cells/well) were exposed to starvation by eliminating serum and growth factor for $24 \mathrm{~h}$ and then added to the top chambers. The bottom chambers were filled with serum-containing medium. Cultures were maintained for $48 \mathrm{~h}$. Cells adherent to the upper surface of the filter were removed using a cotton applicator, and then stained with crystal violet. Cells were counted in 10 random fields at $\times 100$ magification and the mean \pm SD was calculated. To assure a representative conduct of the assays, they were performed in triplicate wells and repeated twice.

Statistical analysis. Statistical analyses were performed using SPSS 18.0 software. Chi-square tests and Fisher's exact tests were used to compare the clinicopathological data. KaplanMeier analysis was used to estimate survival rates and the two-sided log-rank test was used to compare differences. Univariate and multivariate analyses were based on a Cox proportional hazard regression model. In vitro data were analyzed using one-way ANOVA method. A $\mathrm{P}<0.05$ was considered statistically significant.

\section{Results}

PRRX1 and p53 gene expression profiles in HCC. The expression of PRRX1 and p53 were measured in paraffin-embedded serial sections from 116 HCC patients who had undergone hepatectomy. Results showed that the expression of PRRX1 and p53 is downregulated in HCC tissues compared to adjacent liver tissues (Fig. 1A). Furthermore, the expression level of PRRX1 and p53 protein were lower in tumors than that in the corresponding non-malignant liver tissues (Fig. 1B). These results were confirmed by western blot assay with HCC 


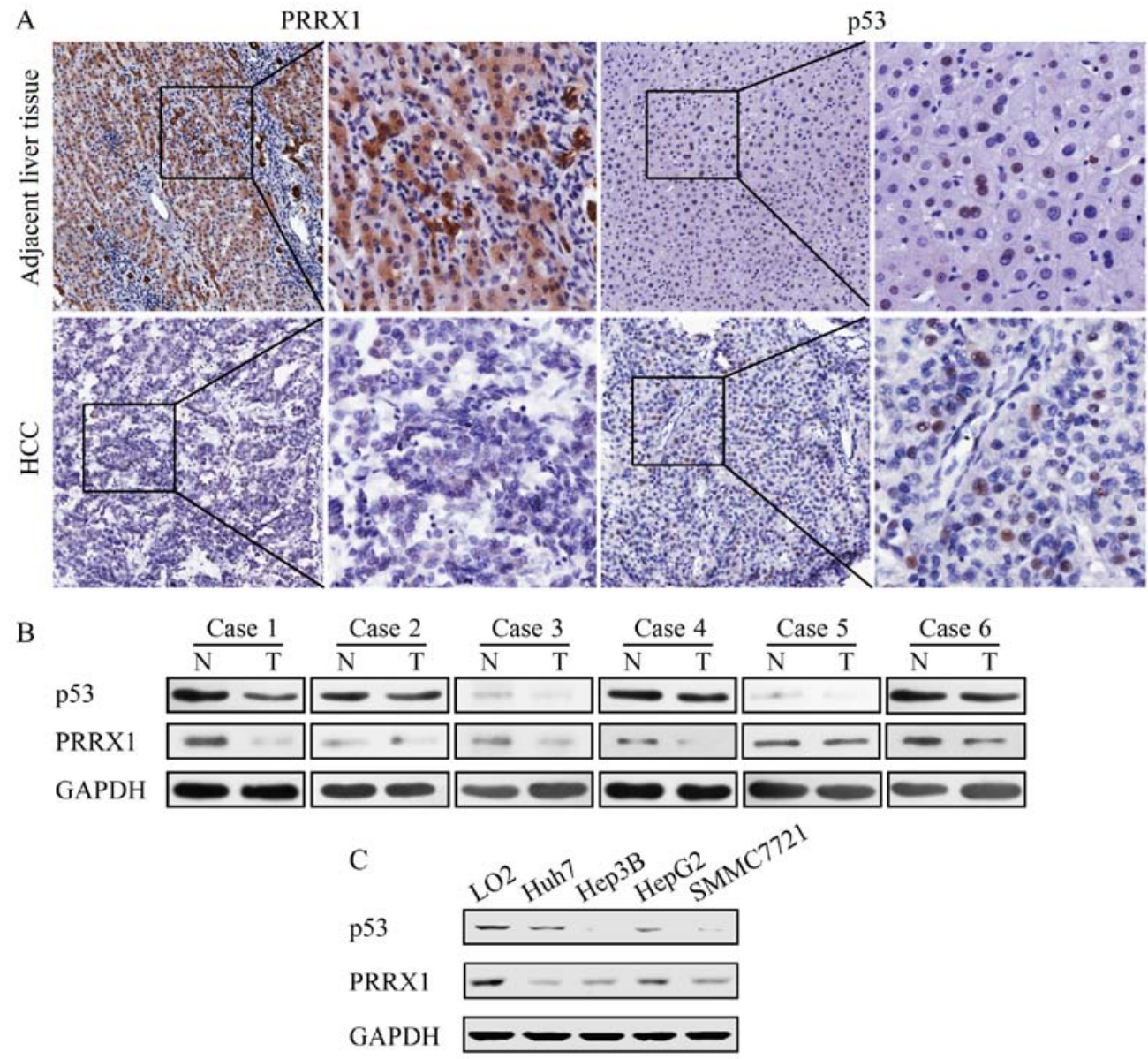

Figure 1. The expression of PRRX1 and p53 in HCC tissues and cell lines. (A) Representative immunohistochemistry images of human HCC tissues for detection of PRRX1 and 553 (magnification, x100). (B) Protein expression of PRRX1 and p53 in HCC tissues and adjacent liver tissues were detected by western blotting. GAPDH was used as a control. (C) Protein expression of PRRX1 and p53 in HCC cell lines and normal liver cells. GAPDH was used as a control.

cell lines including Hep3B (p53 null), Huh7 (p53 mutation), SMMC7721 (p53 wild-type), HepG2 (p53 wild-type) and normal liver cell line LO2 for comparison. The expression of PRRX1 and p53 decreased in all HCC cell lines compared to normal liver cells (Fig. 1C).

The loss of PRRX1 promotes HCC cell mobility in vitro. Western blot assays were used to evaluate the effect of PRRX1 silencing on the expression of p53 in HCC cells (SMMC7721 and HepG2). Our results showed that siRNA silencing of PRRX1 significantly decreased the expression of p53 compared to controls and scrambled groups (Fig. 2A). The decrease of PRRX1 was correlated with downregulation of p53 expression in HCC cells. Transwell and wound healing results showed that PRRX1 siRNA had a stronger promotive effect on cell migration and invasion ability of SMMC7721 and HepG2 cells compared to blank and scrambled group (Fig. 2B and C). Furthermore, HCC cells presented strongest migration and invasion ability when PRRX1 and p53 were both downregulated (Fig. 3). These findings indicated that decreased expression of PRRX1 and/or p53 induced both the migration and the invasion features of HCC cells.

The loss of PRRX1 inhibits HCC cells apoptosis via regulating p53 expression. The Annexin V/PI apoptosis kit was used to quantify the percentage of cells undergoing apoptosis. As shown in Fig. 4, the apoptosis rate of SMMC7721 and HepG2 was $9.18 \pm 2.36$ and $9.40 \pm 3.28 \%$ in response to transfection with PRRX1 siRNA, respectively. The apoptosis of SMMC7721 and HepG2 was $10.65 \pm 3.74$ and $9.24 \pm 2.32 \%$ in response to transfection with p53 siRNA, respectively. Apoptosis of SMMC7721 and HepG2 was $6.65 \pm 2.74$ and $5.24 \pm 3.02 \%$ in response to transfection with both PRRX1 siRNA and p53 siRNA, respectively (Fig. 4). Therefore, silencing PRRX1 and/or p53 exhibited a strong effect on inhibition of apoptosis of HCC cells. In accordance with the observed apoptotic effect induced by PRRX1 siRNA, an anti-apoptotic expression profile was upregulated accompanied by downregulated expression of p53 (Fig. 3A).

Decreased expression of PRRX1 and p53 in HCC is associated with poor prognosis. We first observed a lower PRRX1 and p53 expression in 116 HCC samples (as compared to matched adjacent non-tumor liver tissues (Fig. 1A and B). We additionally found a correlation between the expression level and tumor features. The decreased expression of PRRX1 was found to be significant in HCC patients with vascular invasion $(\mathrm{P}<0.001)$, TNM stage $(\mathrm{P}=0.036)$, $\mathrm{BCLC}$ stage $(\mathrm{P}=0.013)$, intrahepatic $(\mathrm{P}=0.002)$ and distant metastasis $(\mathrm{P}<0.001$; Table I). The decreased expression of p53 was correlated with vascular invasion $(\mathrm{P}<0.001), \mathrm{TNM}$ stage $(\mathrm{P}=0.005), \mathrm{BCLC}$ stage $(\mathrm{P}=0.001)$, intrahepatic $(\mathrm{P}=0.001)$ and distant metastasis 
A
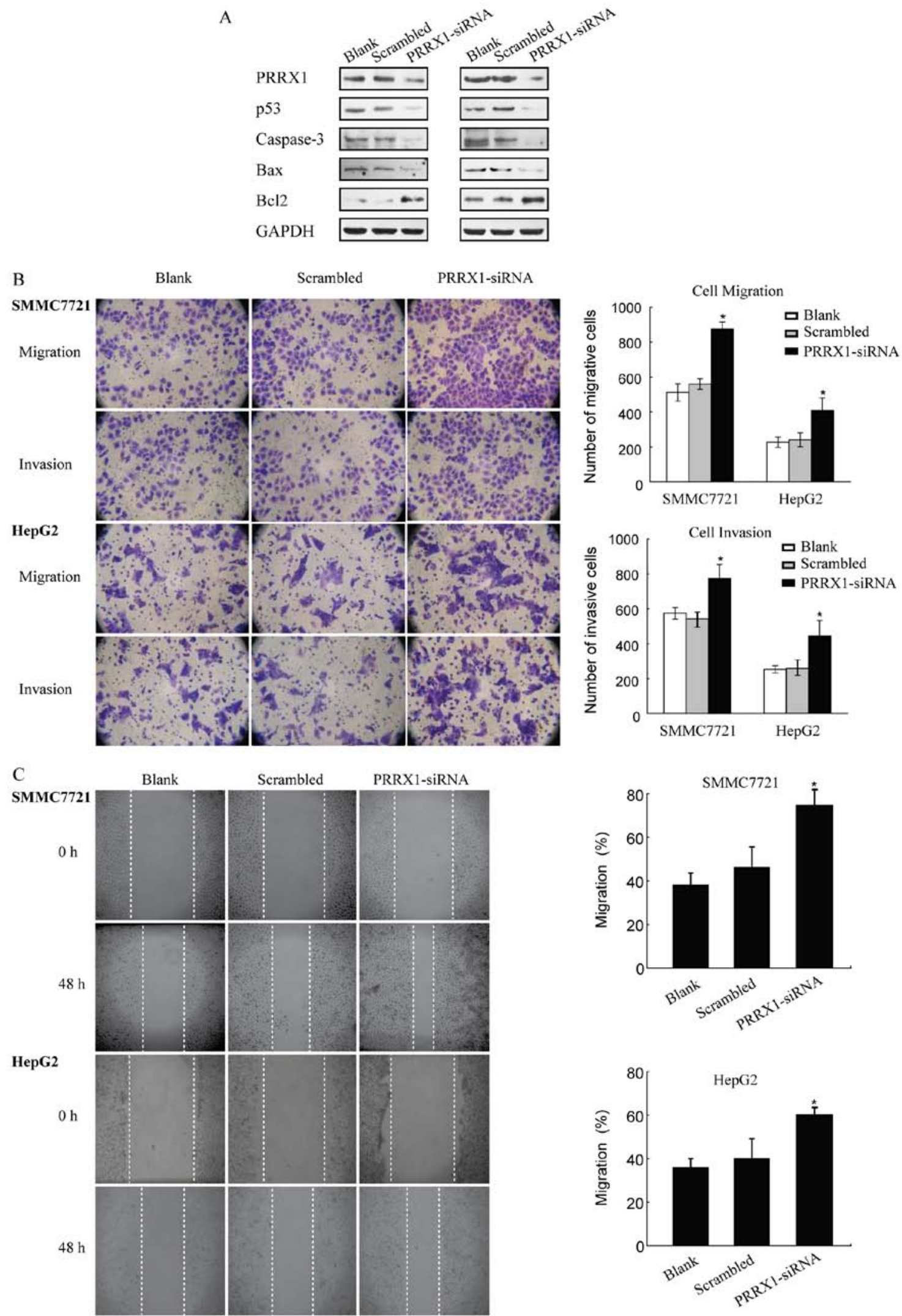

Figure 2. Decreased expression of PRRX1 promotes apoptosis resistance, migration and invasion of HCC cells by regulating p53 expression. (A) Expression of apoptosis-related protein in SMMC-7721 and HepG2 cultured with PRRX1-siRNA, detected by western blotting. GAPDH was used as a control. (B and C) Wound healing assays and Transwell assays show increased migration and invasion ability of HCC cells by using PRRX1-siRNA. The numbers of invasive HCC cells were calculated of ten random microscopic fields. Data are shown as the mean $\pm \mathrm{SD}$ of three independent experiments ("P $<0.05$ ).

$(\mathrm{P}=0.004$; Table I). Based on these results, we divided 116 HCC patients into 4 groups: both high-expression of PRRX1 $(\mathrm{n}=17)$, both low-expression of PRRX1 ( $=23)$, high-expression of PRRX1 and low-expression of p53 $(n=22)$, high-expression 

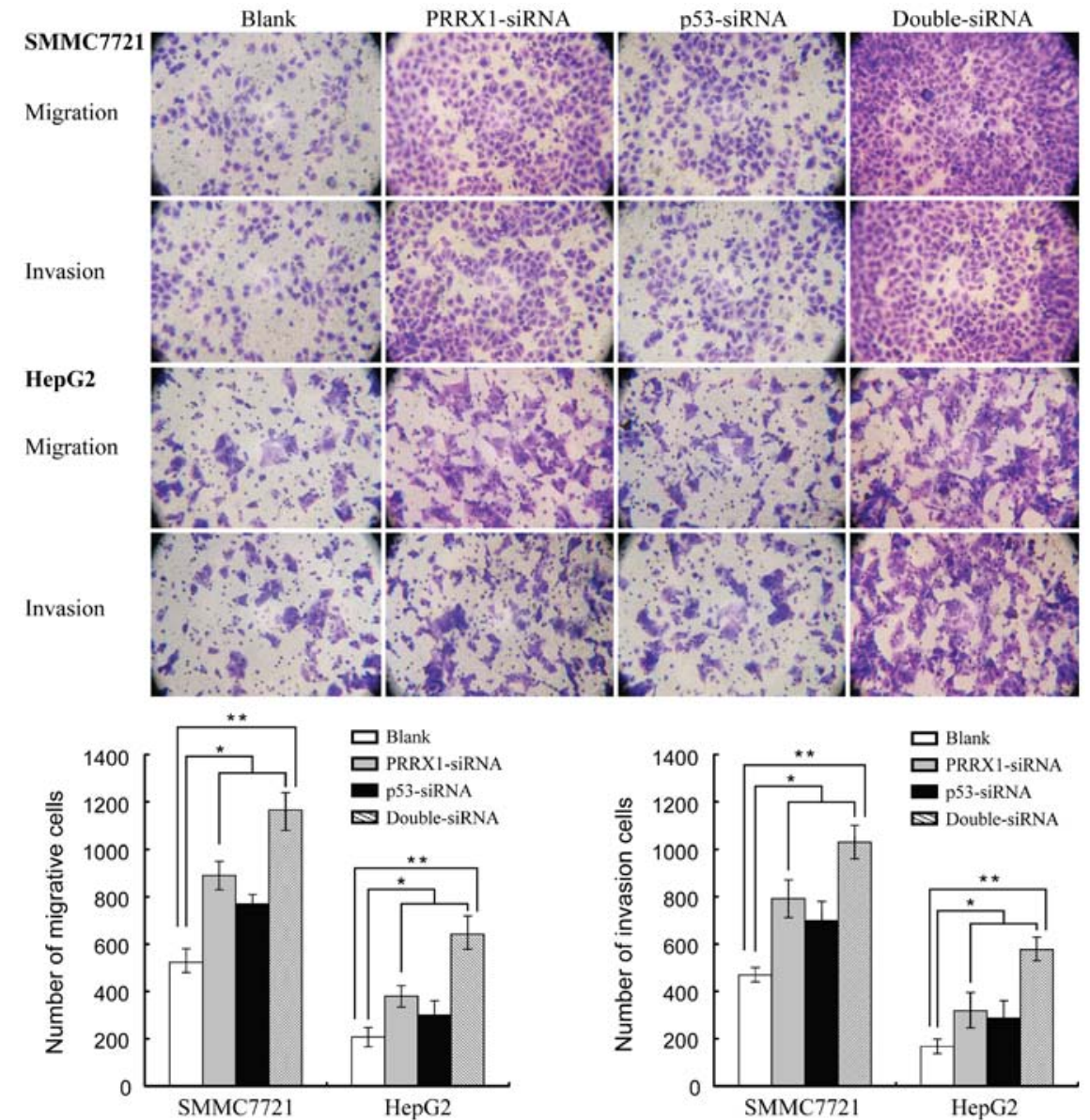

Figure 3. Depletion of PRRX1 and p53 enhances migration and invasion ability of HCC cells. Transwell assays show that downregulation of both PRRX1 and p53 present increased migration and invasion ability of HCC cells compared with PRRX1-siRNA or p53-siRNA alone.
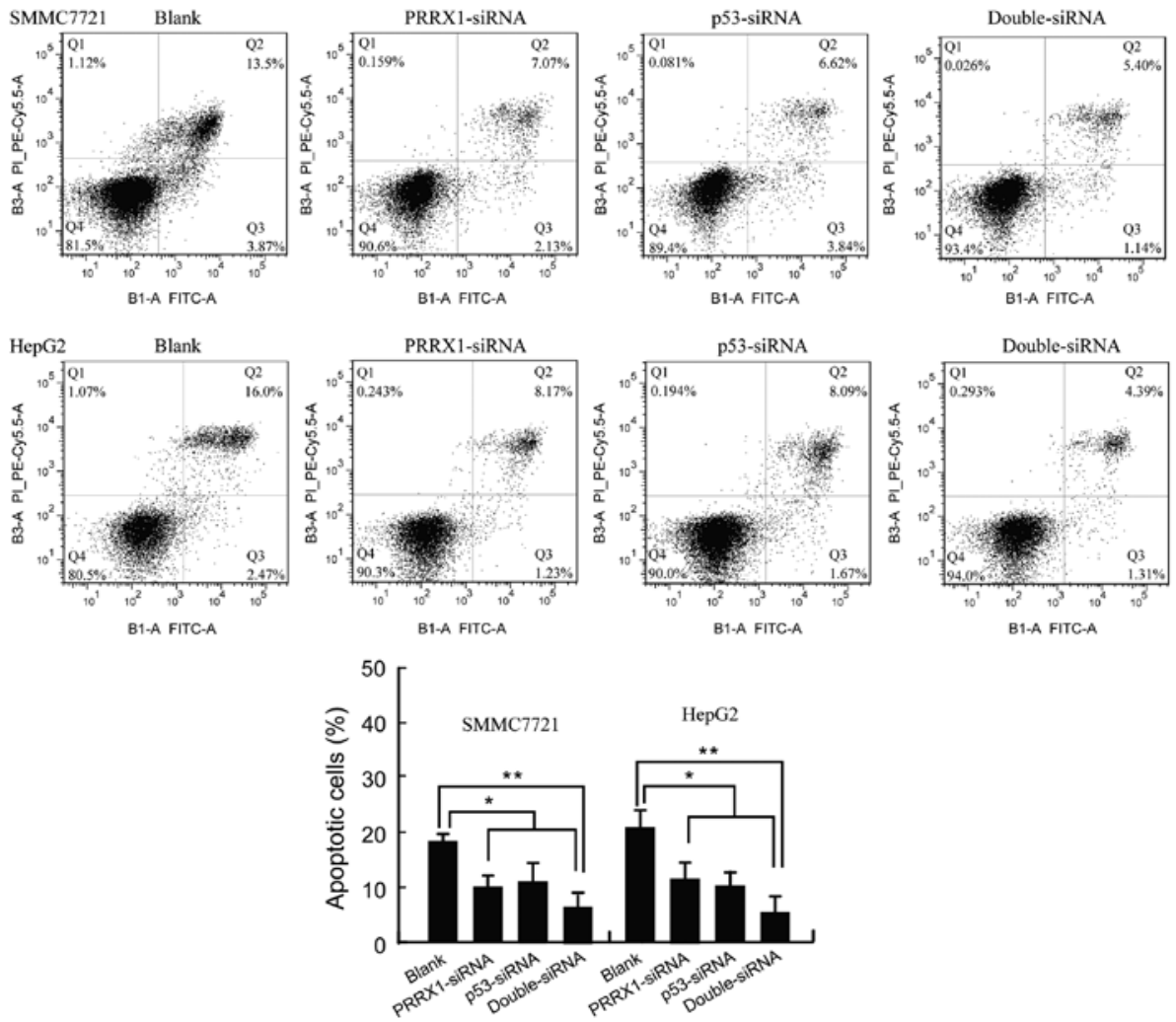

Figure 4. Depletion of PRRX1 and p53 affects HCC cell apoptosis. Interfering with the expression of PRRX1 or p53 inhibits apoptosis of HCC cells. Both decreased expression of PRRX1 and 53 induced stronger resistance to apoptosis of HCC cells ( $\left.{ }^{*} \mathrm{P}<0.05,{ }^{* *} \mathrm{P}<0.01\right)$. 

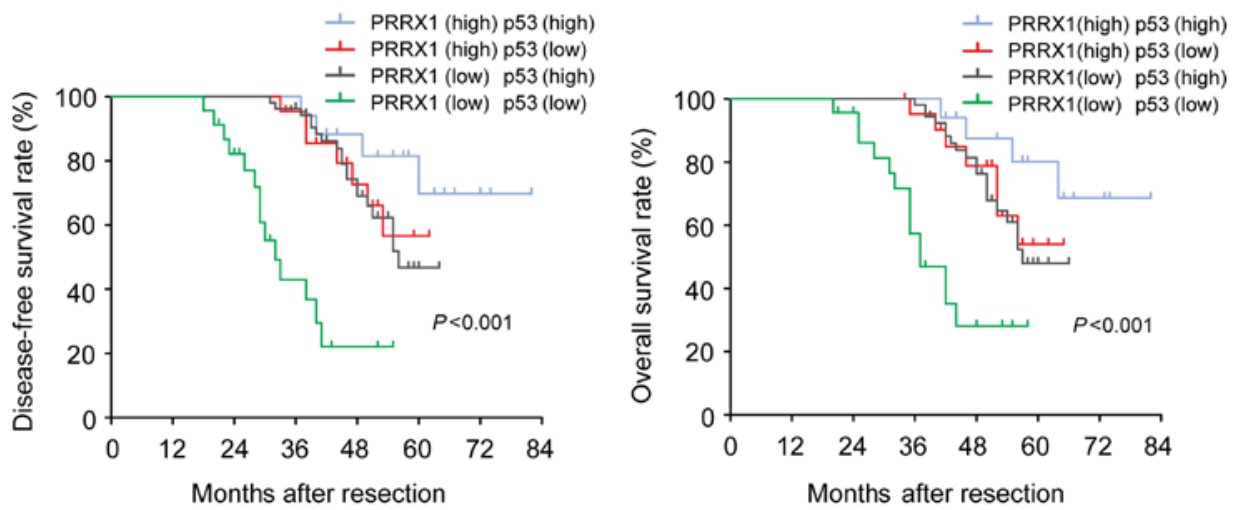

Figure 5. Decreased expression of PRRX1 and p53 is associated with poor prognosis in HCC patients. Overall survival rate and disease-free survival rate in 116 patients who underwent resection for primary HCC based on PRRX1 and p53 expression. Both downregulated expression of PRRX1 and p53 are associated with a poorer overall and disease-free survival rate $(\mathrm{P}<0.001)$.

Table II. Correlation of PRRX1 with p53 in 116 HCC patients.

\begin{tabular}{cccccc}
\hline & \multicolumn{2}{c}{ PRRX1 } & & \\
\cline { 2 - 3 } & Low & High & & r & P-value \\
\hline p53 & & & & \\
Low & 23 & 22 & 0.257 & 0.006 \\
High & 54 & 17 & & \\
\hline
\end{tabular}

of p53 and low-expression of PRRX1 (n=54). HCC patients with low-expression of both PRRX1 and p53 had a significantly shorter overall and disease-free survival than patients with only PRRX1- or only p53 high expression (Fig. 5). Their correlations are detailed in Table II, and PRRX1 is positively correlated with $\mathrm{p} 53$ expression $(\mathrm{r}=0.257, \mathrm{P}=0.006)$. These observations are suggestive that PRRX1 and $\mathrm{p} 53$ expression levels could be valuable predictive factors for recurrence and survival in patients with HCC. Co-downregulation of both PRRX1 and p53 was confirmed to be an independent negative factor for overall and diseased-free survival.

\section{Discussion}

Hepatocellular carcinoma (HCC) is a common malignancy worldwide, especially occurring in Asia and South Africa. The incidence of HCC in China is still high. Molecular mechanisms leading to malignant transformation of normal liver cells have not yet been fully elucidated. PRRX1 is a transcription co-activator with the function of enhancing the DNA-binding activity of serum response factor. It also regulates muscle creatine kinase, indicating a role in the establishment of diverse mesodermal muscle types. Several recent studies demonstrate that PRRX1 can regulate differentiation of mesenchymal precursors. Ocaña et al (11) showed that PRRX1 is an EMT inducer conferring migratory and invasive properties. Hirata et al (14) found that downregulation of PRRX1 expression contributes to poor prognosis of patients with HCC through acquisition of CSC-like properties. The loss of PRRX1 is required for breast cancer cells and HCC cells to metastasize in vivo. In contrast to studies of breast cancer, overexpression of PRRX1 was significantly associated with metastasis and poor prognosis in CRC (12). It indicates that heterogeneity exists in different tumors. The present study demonstrated that PRRX1 expression is lower in HCC tissues than adjacent normal liver tissues and is significantly correlated with the survival and metastasis of HCC cells in vitro. The mechanism underlying PRRX1 expression and HCC remains unclear.

The tumor suppressor p53 is a transcription factor that responds to various types of cellular stress, such as oncogene activation and genotoxic drug-induced DNA damage (21). p53 regulates a variety of cellular behaviors, such as cell growth, DNA repair, cell cycle arrest and apoptosis (15). Wild-type p53 gene mutation and inactivation in liver cells leading by a variety of environmental factors play an important role in carcinogenesis. When the cell genome DNA was damaged by exogenous factors, p53 will build a complex regulatory network with related genes and regulate cell characteristics by 553 -related signaling pathway.

In this study, the expression of PRRX1 and p53 were found decreased in some HCC cell lines and clinical samples. Moreover, we found that p53 expression was correlated with PRRX1 expression in HCC. siRNA silencing of PRRX1 significantly decreased the expression of p53 in HepG2 and SMMC7721. Our results indicated that downregulation of PRRX1 expression in HCC cells presenting more aggressive cellular motility. It is reported that p53 participates in inducing apoptosis in HCC cells (22-24). Our data revealed that silencing PRRX1 exhibited a stronger effect on inhibiting apoptosis via regulating p53 expression of HCC cells. Furthermore, we demonstrated that decreased expression of PRRX1 and p53 was significantly associated with poor prognosis in patients with HCC.

In summary, we report that PRRX1 regulates p53 by inhibiting apoptosis in HCC cells. The loss of PRRX1 expression stimulates invasion and metastasis of HCC cells, contributing to poor prognosis. Our results concerning the relationship between PRRX1 expression and p53 expression suggest that HCC patients who have both low expression of PRRX1 and p53 are more likely to develop metastases and have the worst prognosis, and this knowledge can be used to predict patient outcomes. Our finding suggested that 
PRRX1 and p53 synergistically inhibit HCC progression and metastasis by inducing apoptosis. Further experiments are necessary to determine whether they have a positive effect on HCC therapy.

\section{References}

1. Bosch FX, Ribes J, Díaz M and Cléries R: Primary liver cancer: Worldwide incidence and trends. Gastroenterology 127 (Suppl 1): S5-S16, 2004.

2. Yuen MF, Tanaka Y, Fong DY, Fung J, Wong DK, Yuen JC, But DY, Chan AO, Wong BC, Mizokami M, et al: Independent risk factors and predictive score for the development of hepatocellular carcinoma in chronic hepatitis B. J Hepatol 50: 80-88, 2009.

3. Tsai JH, Donaher JL, Murphy DA, Chau S and Yang J: Spatiotemporal regulation of epithelial-mesenchymal transition is essential for squamous cell carcinoma metastasis. Cancer Cell 22: 725-736, 2012 .

4. Tsai JH and Yang J: Epithelial-mesenchymal plasticity in carcinoma metastasis. Genes Dev 27: 2192-2206, 2013.

5. Bae YK, Choi JE, Kang SH and Lee SJ: Epithelial-mesenchymal transition phenotype is associated with clinicopathological factors that indicate aggressive biological behavior and poor clinical outcomes in invasive breast cancer. J Breast Cancer 18 256-263, 2015

6. Polyak K and Weinberg RA: Transitions between epithelial and mesenchymal states: Acquisition of malignant and stem cell traits. Nat Rev Cancer 9: 265-273, 2009.

7. Taneyhill LA, Coles EG and Bronner-Fraser M: Snail2 directly represses cadherin 6B during epithelial-to-mesenchymal transitions of the neural crest. Development 134: 1481-1490, 2007.

8. Deep G, Jain AK, Ramteke A, Ting H, Vijendra KC, Gangar SC, Agarwal C and Agarwal R: SNAI1 is critical for the aggressiveness of prostate cancer cells with low E-cadherin. Mol Cancer. 13: 37, 2014.

9. Campbell K, Whissell G, Franch-Marro X, Batlle E and Casanova J: Specific GATA factors act as conserved inducers of an endodermal-EMT. Dev Cell 21: 1051-1061, 2011.

10. Song K, Li Q, Jiang ZZ, Guo CW and Li P: Heparan sulfate D-glucosaminyl 3-O-sulfotransferase-3B1, a novel epithelialmesenchymal transition inducer in pancreatic cancer. Cancer Biol Ther 12: 388-398, 2011.

11. Ocaña OH, Córcoles R, Fabra A, Moreno-Bueno G, Acloque H, Vega S, Barrallo-Gimeno A, Cano A and Nieto MA: Metastatic colonization requires the repression of the epithelial-mesenchymal transition inducer Prrx1. Cancer Cell 22: 709-724, 2012.
12. Takahashi Y, Sawada G, Kurashige J, Uchi R, Matsumura T, Ueo H, Takano Y, Akiyoshi S, Eguchi H, Sudo T, et al: Paired related homoeobox 1, a new EMT inducer, is involved in metastasis and poor prognosis in colorectal cancer. Br J Cancer 109: 307-311, 2013.

13. Guo J, Fu Z, Wei J, Lu W, Feng J and Zhang S: PRRX1 promotes epithelial-mesenchymal transition through the Wnt/ $\beta$-catenin pathway in gastric cancer. Med Oncol 32: 393, 2015.

14. Hirata H, Sugimachi K, Takahashi Y, Ueda M, Sakimura S, Uchi R, Kurashige J, Takano Y, Nanbara S, Komatsu H, et al: Downregulation of PRRX1 confers cancer stem cell-like properties and predicts poor prognosis in hepatocellular carcinoma. Ann Surg Oncol 22 (Suppl 3): S1402-S1409, 2015

15. Vogelstein B, Lane D and Levine AJ: Surfing the p53 network. Nature 408: 307-310, 2000

16. Chari NS, Pinaire NL, Thorpe L, Medeiros LJ, Routbort MJ and McDonnell TJ: The p53 tumor suppressor network in cancer and the therapeutic modulation of cell death. Apoptosis 14: 336-347, 2009.

17. Soussi T: $\mathrm{p} 53$ alterations in human cancer: More questions than answers. Oncogene 26: 2145-2156, 2007.

18. Lewis BC, Klimstra DS, Socci ND, Xu S, Koutcher JA and Varmus HE: The absence of $\mathrm{p} 53$ promotes metastasis in a novel somatic mouse model for hepatocellular carcinoma. Mol Cell Biol 25: 1228-1237, 2005.

19. Chen YW, Klimstra DS, Mongeau ME, Tatem JL, Boyartchuk V and Lewis BC: Loss of p53 and Ink4a/Arf cooperate in a cell autonomous fashion to induce metastasis of hepatocellular carcinoma cells. Cancer Res 67: 7589-7596, 2007.

20. Hansen JE, Fischer LK, Chan G, Chang SS, Baldwin SW, Aragon RJ, Carter JJ, Lilly M, Nishimura RN, Weisbart RH, et al: Antibody-mediated p53 protein therapy prevents liver metastasis in vivo. Cancer Res 67: 1769-1774, 2007.

21. Marte B: Cancer: Super p53. Nature 420: 279, 2002.

22. Yee SB, Choi HJ, Chung SW, Park DH, Sung B, Chung HY and Kim ND: Growth inhibition of luteolin on HepG2 cells is induced via 553 and Fas/Fas-ligand besides the TGF- $\beta$ pathway. Int J Oncol 47: 747-754, 2015.

23. Zhu R, Mok MT, Kang W, Lau SS, Yip WK, Chen Y, Lai PB, Wong VW, To KF, Sung JJ, et al: Truncated HBx-dependent silencing of GAS2 promotes hepatocarcinogenesis through deregulation of cell cycle, senescence and p53-mediated apoptosis. J Pathol 237: 38-49, 2015.

24. Lou G, Liu Y, Wu S, Xue J, Yang F, Fu H, Zheng M and Chen Z: The p53/miR-34a/SIRT1 positive feedback loop in quercetininduced apoptosis. Cell Physiol Biochem 35: 2192-2202, 2015. 